\title{
Equivalence Theory Applied to Anisotropic Thin Plates
}

\author{
Madjid Haddad, Yves Gourinat, Miguel Charlotte \\ Université de Toulouse, INSA, UPS, Mines d'Albi, ISAE, Institut Clément ADER (ICA), Toulouse, France \\ E-mail: madjid.haddad@isae.fr \\ Received October 18, 2010; revised June 10, 2011; accepted June 20, 2011
}

\begin{abstract}
We extend the Equivalence Theory (ET) formulated by Absi [1] for the statics of isotropic materials to the statics and dynamics of orthotropic materials. That theory relies on the assumption that any real body modeling may be substituted by another one that, even though it may possibly have material constitutive laws and geomet- ric properties with no physical sense (like negative cross sections or Young modulus), is intended to be more advantageous for calculus. In our approach, the equivalence is expressed by equating both the effective strain energies of the two models and the material structural weights in dynamics [2]. We provide a numerical analysis of the convergence properties of ET approach while comparing its numerical results with those pre- dicted by the analytical theory and the Finite Elements Method for thin plates.
\end{abstract}

Keywords: Equivalence Theory, Thin Plates, Anisotropic Plates, Dynamics

\section{Introduction}

Around the 70s some researchers $[1,3]$ were interested in the problem of modelling a slab as a beam lattice or truss. Such a substitution relies on some common engineering viewpoint according which if the slab can be subdivided into slices parallel to its boundaries (like $\mathrm{AB}$ or $\mathrm{CD}$ on Figures 1 and 2), then each slice may be assimilated to a beam with related material and geometrical properties. This coarse structure approach which is suitable for pre-sizing was also assumed to be applicable to the calculation of arch dams (Figure 3) sliced into arcs (AB) and consoles $(\mathrm{CD})$.

Such a practical problem gave birth to the Equivalence Theory (ET) developed by Absi [1] who specified the general conditions of equivalence in statics for isotropic materials. In statics, the standard equivalence criterion is expressed between the strain energies. That theory assumes that any real body modelling may be substituted by another one that, even though it may possibly have material constitutive laws and geometric properties with no physical meanings (like negative cross sections or Young modulus), is intended to be more advantageous for calculus. In the literature $[1,2,4]$, only few investigations have tried to check this assumption. These equivalence analyses were formulated for the statics of different cases of isotropic slabs and the comparisons made with the analytical theory and the Finite Element Method (FEM) analysis have led to very encouraging conclusions.
However, the ET has been abandoned in favour of the FEM because the latter is more flexible to deal with structures with arbitrary geometry.

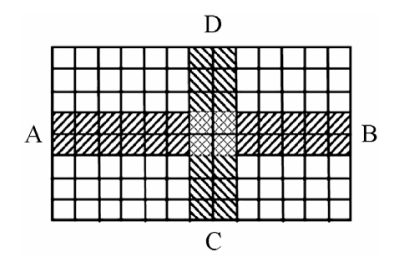

Figure 1. Rectangular slab subdivided into slices parallel to its boundaries.

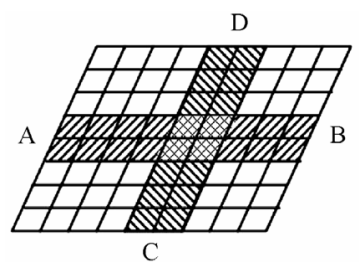

Figure 2. Lozenge slab subdivided into slices parallel to its boundaries.

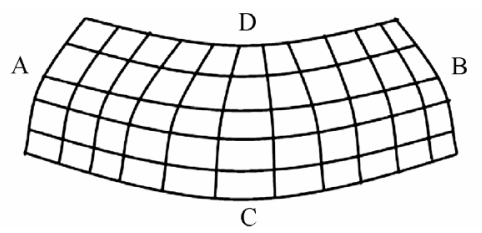

Figure 3. Arch dam subdivided into arcs. 
This work aims to extend the ET approach to the analysis of anisotropic plates subjected to transverse loads, in statics, and to study the frequencies and the modes shapes in dynamics. To check the validity of the extended formulation, numerical comparisons with analytical solutions in statics and to FEM results in dynamics are made. As mentioned previously, the possible lack of meaning of the resulting equivalent model makes the treatment of such a method on commercial FEM software (Patran/Nastran, Catia...) difficult, if not impossible. Therefore in order to compare our new improvement of the theory and overcome these difficulties, we were led to develop a finite element computer codes in Matlab.

\section{Formulation of the Method in Statics}

The Equivalence Theory (ET) aims at replacing a given real body by another arbitrary chosen, and possibly fictitious, one [1]. The standard equivalence criterion is expressed between the strain energies. Here we will choose the substituting body as a lattice structure with beams elements. We will first express the energy of tensile and bending of an anisotropic plate. Then, according with the ET, we will identify the cross sections, the quadratic moments and the central moments of inertia of beams, by making a comparison between the Elementary Representative Cells (ERC) of the two models.

\subsection{Expressing Equivalence in Traction}

\subsubsection{Elastic Energy of an Orthotropic Plate: [4]}

Consider the case of a thin, linearly elastic and anisotropic plate, with three planes of symmetry and a constant thickness $h$. The plate stress strain relations are written in this case as follows:

$$
\begin{aligned}
& \sigma_{x}=E_{x}^{\prime} \varepsilon_{x}+E^{\prime \prime \prime} \varepsilon_{y} ; \\
& \sigma_{y}=E_{y}^{\prime} \varepsilon_{y}+E^{\prime \prime \prime} \varepsilon_{x} ; \tau_{x y}=2 G \gamma_{x y}
\end{aligned}
$$

with $E_{x}^{\prime}=k E_{l} ; E_{y}^{\prime}=k E_{t} ; \quad E^{\prime \prime}=k v_{t l} E_{l}=k v_{l t} E_{t} ; \quad G=G_{l t}$

Hereabove $\sigma_{x}, \sigma_{y}, \tau_{x y}$ denote respectively the normal stresses with respect to $x$ and $y$ axes, and the shear stress, while

$$
\varepsilon_{x}=\frac{\partial u}{\partial x} ; \varepsilon_{y}=\frac{\partial v}{\partial x} ; \gamma_{x y}=\frac{1}{2}\left(\frac{\partial u}{\partial x}+\frac{\partial v}{\partial y}\right)
$$

are respectively the related normal and shear strains; besides $E_{l}$ and $E_{t}$ : are respectively the longitudinal and transversal Young's moduli, $v_{l t}$ and $v_{t l}=v_{l t} \frac{E_{t}}{E_{l}}$ are the longitudinal and transversal Poisson's ratios, which provide $k=\frac{1}{1-\left(v_{l t} v_{t l}\right)}$, and $G_{l t}$ is the Coulomb's coefficient.
Suppose now that the plate is subjected to a uniform bi-axial tensile along the $\mathrm{x}$ and $\mathrm{y}$ axis. Hence its elastic energy reads like

$$
U_{t}=\frac{A_{p}}{2}\left(E_{x}^{\prime} \varepsilon_{x}^{2}+E_{y}^{\prime} \varepsilon_{y}^{2}+2 E^{\prime \prime} \varepsilon_{x} \varepsilon_{y}+2 G \gamma_{x y}{ }^{2}\right)
$$

where $A_{p}$ represents the ERC surface area of the plate while noticing that the strains are independent on $x, y$ or $z$. Note that we recover the traction energy of a materially isotropic plates in plane stress for $E=E_{t}=E_{l}$ (the same Young's modulus) and $v=v_{t l}=v_{l t}$ (same Poisson's ratio).

\subsubsection{Beam Traction Energy}

The traction energie of a beam " $i j$ ", Figure 4 is:

$$
W_{i j}=\rho_{i j}\left[e_{11} \cos ^{2} \theta+e_{22} \sin ^{2} \theta+2 e_{12} \sin \theta \cos \theta\right]^{2}
$$

where $\rho_{i j}=E S_{i j} l_{e}$ is a characteristic traction parameter, while $S_{i j}, l_{e}$ and $E$ are respectively the cross section, length and Young's modulus of the beam.

\subsubsection{Expressing the Traction Equivalence}

The equivalence is expressed in an elementary representative cell ERC of the two structures.

Consider the cell represented in Figure 5.

The strain energy expressions of the different beams constituting this cell are:

$$
\begin{aligned}
W_{A B}^{\prime} & =W_{C D}^{\prime}=\frac{1}{2} \rho_{A B}\left(e_{11}\right)^{2} \\
W_{A C}^{\prime} & =W_{B D}^{\prime}=\frac{1}{2} \rho_{A C}\left(e_{22}\right)^{2} \\
W_{A D}^{\prime} & =\frac{1}{2} \rho_{A D}\left[e_{11} \cos ^{2} \alpha+e_{22} \sin ^{2} \alpha+2 e_{12} \sin \alpha \cos \alpha\right]^{2} \\
W_{B C}^{\prime} & =\frac{1}{2} \rho_{B D}\left[e_{11} \cos ^{2} \alpha+e_{22} \sin ^{2} \alpha-2 e_{12} \sin \alpha \cos \alpha\right]^{2}
\end{aligned}
$$

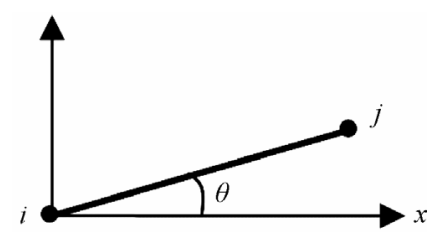

Figure 4. A beam representation in the plane.

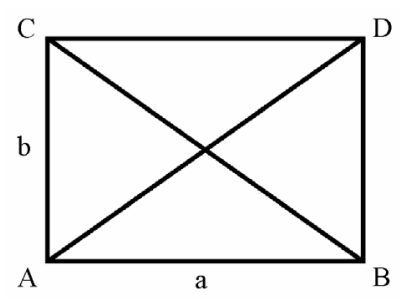

Figure 5. ERC “square with diagonals”. 
Write the equivalence between the two cells, we have then:

$$
\begin{aligned}
U_{t}= & \frac{A_{p}}{2}\left(E_{X}^{\prime}\left(e_{11}\right)^{2}+E_{y}^{\prime}\left(e_{22}\right)^{2}+2 E^{\prime \prime} e_{11} e_{22}+2 G\left(e_{12}\right)^{2}\right) a \\
= & \rho_{A B}\left(e_{11}\right)^{2}+\rho_{A C}\left(e_{22}\right)^{2} \\
& +\frac{1}{2} \rho_{A D}\left[e_{11} \cos ^{2} \alpha+e_{22} \sin ^{2} \alpha+2 e_{12} \sin \alpha \cos \alpha\right]^{2} \\
& +\frac{1}{2} \rho_{B D}\left[e_{11} \cos ^{2} \alpha+e_{22} \sin ^{2} \alpha-2 e_{12} \sin \alpha \cos \alpha\right]^{2}
\end{aligned}
$$

Taking $\rho_{A D}=\rho_{B D}$, and comparing the two terms of the Equation (6) we can write:

$$
\begin{aligned}
\frac{A_{p} E_{x}^{\prime}}{2}\left(e_{11}\right)^{2}= & \rho_{A B}\left(e_{11}\right)^{2}+\rho_{A D}\left(e_{11}\right)^{2} \cos ^{4} \alpha \\
\rho_{A B} & =\frac{A_{p} E_{x}^{\prime}}{2}-\rho_{A D} \cos ^{4} \alpha \\
\frac{A_{p} E_{y}^{\prime}}{2}\left(e_{22}\right)^{2}= & \rho_{A C}\left(e_{22}\right)^{2}+\rho_{A D}\left(e_{22}\right)^{2} \sin ^{4} \alpha \\
\rho_{A C} & =\frac{A_{p} E_{y}^{\prime}}{2}-\rho_{A D} \sin ^{4} \alpha \\
A_{p} G\left(e_{12}\right)^{2} & =4 \rho_{A D} \sin ^{2} \alpha \cos ^{2} \alpha\left(e_{12}\right)^{2} \\
\rho_{A D} & =\frac{A_{p} G}{4} \frac{1}{\sin ^{2} \alpha \cos ^{2} \alpha} \\
A_{p} E^{\prime \prime} e_{11} e_{22} & =2 \rho_{A D} \sin ^{2} \alpha \cos ^{2} \alpha\left(e_{11} e_{22}\right) \\
\rho_{A D} & =\frac{A_{p} E^{\prime \prime}}{2} \frac{1}{\sin ^{2} \alpha \cos ^{2} \alpha}
\end{aligned}
$$
tain:

Replacing $\rho_{A D}$ in the Equations (7) and (8), we ob-

$$
\begin{gathered}
\rho_{A B}=k \frac{A_{p} E_{l}}{2}\left(1-v_{t l} \cot ^{2} \alpha\right) \\
\rho_{A C}=k \frac{A_{p} E_{t}}{2}\left(1-v_{l t} \tan ^{2} \alpha\right) \\
\rho_{A D}=\frac{A_{p} E_{l}}{2} \frac{v_{t l}}{\sin ^{2} \alpha \cos ^{2} \alpha}
\end{gathered}
$$

We know that: $\rho_{A B}=(E A)_{A B} a, \rho_{A C}=(E A)_{A C} b$ and $\rho_{A D}=(E A)_{A D} l$;

where $A_{i j}, E_{i j}$ are respectively the cross section and the Young's modulus of the beam " $i j$ " and $a, b$, l: lengths of the considered beam, as shown in Figure 5.

Let's consider that the beams which are parallel to the $\mathrm{x}$ axis and the diagonal ones have the same Young's modulus as the longitudinal one of the plate, and those which are parallel to the y axis, have the same Young's modulus as the transverse one of the plate.

Finally, we obtain:

$$
\begin{gathered}
A_{A B}=k \frac{b h}{2}\left(1-v_{l t} \cot ^{2} \alpha\right) ; \\
A_{A C}=k \frac{a h}{2}\left(1-v_{l t} \tan ^{2} \alpha\right) ; \\
A_{A D}=k \frac{a b h}{2 l}\left(\frac{v_{l t}}{\sin ^{2} \alpha \cos ^{2} \alpha}\right) .
\end{gathered}
$$

\subsection{Bending Equivalence}

\subsubsection{Plate Bending Energy}

In our study we consider the Kirchoff-Love's plates, which suppose that the straight linear elements that are perpendicular to the plate's mean surface still remain so even after deformation.

We have:

$$
\varepsilon_{x}=-z \frac{\partial^{2} w}{\partial x^{2}} ; \varepsilon_{y}=-z \frac{\partial^{2} w}{\partial y^{2}} ; \gamma_{x y}=-z \frac{\partial^{2} w}{\partial x \partial y} .
$$

Then the bending and tensional moments are given as follows:

$$
\begin{aligned}
& M_{x}=\int_{-h / 2}^{h / 2} \sigma_{x} z \mathrm{~d} z=-\left(D_{x} \frac{\partial^{2} w}{\partial x^{2}}+D_{1} \frac{\partial^{2} w}{\partial y^{2}}\right) \\
& M_{y}=\int_{-h / 2}^{h / 2} \sigma_{y} z \mathrm{~d} z=-\left(D_{y} \frac{\partial^{2} w}{\partial y^{2}}+D_{1} \frac{\partial^{2} w}{\partial x^{2}}\right) \\
& M_{x y}=\int_{-h / 2}^{h / 2} \tau_{x y} z \mathrm{~d} z=2 D_{x y} \frac{\partial^{2} w}{\partial x \partial y}
\end{aligned}
$$

where $D_{x}=\frac{E_{x}^{\prime} h^{3}}{12} ; D_{y}=\frac{E_{y}^{\prime} h^{3}}{12} ; D_{1}=\frac{E^{\prime \prime} h^{3}}{12} ; D_{x y}=\frac{G h^{3}}{12}$.

The bending energy of the plate is given [5]:

$$
\mathrm{d} V=\frac{1}{2}\left\{-\left(M_{x} \frac{\partial w}{\partial x^{2}}+M_{y} \frac{\partial w}{\partial y^{2}}\right)+\left(M_{x y} \frac{\partial w}{\partial x \partial y}\right)\right\} \mathrm{d} x \mathrm{~d} y,
$$

which gives:

$$
\begin{aligned}
\mathrm{d} V= & \frac{1}{2}\left[D_{x}\left(\frac{\partial w}{\partial x^{2}}\right)^{2}+D_{y}\left(\frac{\partial w}{\partial y^{2}}\right)^{2}\right. \\
& \left.+D_{1}\left(\frac{\partial w}{\partial x^{2}} \frac{\partial w}{\partial y^{2}}\right)+2 D_{x y}\left(\frac{\partial w}{\partial x \partial y}\right)^{2}\right] \mathrm{d} x \mathrm{~d} y
\end{aligned}
$$

with:

$$
D_{x}=\frac{k E_{l} h^{3}}{12} ; D_{y}=\frac{k E_{t} h^{3}}{12} ; D_{1}=\frac{k v_{t l} E_{l} h^{3}}{12} ; D_{x y}=\frac{G_{l t} h^{3}}{12} .
$$




\subsubsection{Bending and Torsional Energie of a Beam}

The bending and tensional energy contributions of a beam ' $i j$ ' (see $[1,5])$ are respectively given as

$$
\begin{aligned}
W_{f}= & \frac{1}{2} \rho_{i j}^{\prime}\left[\frac{\partial^{2} w}{\partial x^{2}} \cos ^{2} \theta+\frac{\partial^{2} w}{\partial y^{2}} \sin ^{2} \theta\right. \\
& \left.+\frac{\partial^{2} w}{\partial x \partial y} \cos \theta \sin \theta\right]^{2}
\end{aligned}
$$

where $\rho_{i j}^{\prime}=\left(E I l_{e}\right)_{i j}$ is defined with the quadratic moment $I$ of the cross section $S_{i j}$ and

$$
W_{t}=\frac{1}{2} \gamma\left[\frac{1}{2}\left(\frac{\partial^{2} w}{\partial y^{2}}-\frac{\partial^{2} w}{\partial x^{2}}\right) \sin 2 \theta+\frac{\partial^{2} w}{\partial x \partial y} \cos 2 \theta\right]^{2}
$$

where $\gamma=G J l_{e}$.

\subsubsection{Expressing the Bending Equivalence}

Here we will assume that all the beams work in bending and only the longitudinal and diagonal ones work in torsion (Figure 6). This supposition has no effect in the theory itself, since the unique condition to satisfy is to conserve the total strain energy. We can write then:

$$
\begin{gathered}
W_{f A B}=W_{f C D}=\frac{1}{2} \rho_{A B}^{\prime}\left(\frac{\partial^{2} w}{\partial x^{2}}\right)^{2}+\frac{1}{2} \gamma_{A B}\left(\frac{\partial^{2} w}{\partial x \partial y}\right)^{2} \\
W_{f A C}=W_{f B S}=\frac{1}{2} \rho_{A C}^{\prime}\left(\frac{\partial^{2} w}{\partial y^{2}}\right)^{2}+\frac{1}{2} \gamma_{A C}\left(\frac{\partial^{2} w}{\partial x \partial y}\right)^{2} \\
W_{f A D}=\frac{1}{2} \rho_{A D}^{\prime}\left[\frac{\partial^{2} w}{\partial x^{2}} \cos ^{2} \alpha+\frac{\partial^{2} w}{\partial y^{2}} \sin ^{2} \alpha+\frac{\partial^{2} w}{\partial x \partial y} \cos \alpha \sin \alpha\right]^{2} \\
W_{f B C}=\frac{1}{2} \rho_{B C}^{\prime}\left[\frac{\partial^{2} w}{\partial x^{2}} \cos ^{2} \alpha+\frac{\partial^{2} w}{\partial y^{2}} \sin ^{2} \alpha-\frac{\partial^{2} w}{\partial x^{2} y} \cos \alpha \sin \alpha\right]^{2}
\end{gathered}
$$

The equivalence is given by equating the two strain energies:

$$
\begin{aligned}
& W=S V=2 W_{f A B}+W_{f B C}+2 W_{f A C}+W_{f A D}+2 W_{t A B}+2 W_{t A C} \\
& \frac{S}{2}\left[D_{x}\left(\frac{\partial w}{\partial x^{2}}\right)^{2}+D_{y}\left(\frac{\partial w}{\partial y^{2}}\right)^{2}+D_{1}\left(\frac{\partial w}{\partial x^{2}} \frac{\partial w}{\partial y^{2}}\right)+2 D_{x y}\left(\frac{\partial w}{\partial x \partial y}\right)^{2}\right]=\rho_{A B}^{\prime}\left(\frac{\partial^{2} w}{\partial x^{2}}\right)^{2}+\rho_{A C}^{\prime}\left(\frac{\partial^{2} w}{\partial y^{2}}\right)^{2}+ \\
& \frac{1}{2} \rho_{A D}^{\prime}\left[\frac{\partial^{2} w}{\partial x^{2}} \cos ^{2} \alpha+\frac{\partial^{2} w}{\partial y^{2}} \sin ^{2} \alpha+2 \frac{\partial^{2} w}{\partial x \partial y} \sin \alpha \cos \alpha\right]^{2}+\frac{1}{2} \rho_{B D}^{\prime}\left[\frac{\partial^{2} w}{\partial x^{2}} \cos ^{2} \alpha+\frac{\partial^{2} w}{\partial y^{2}} \sin ^{2} \alpha-2 \frac{\partial^{2} w}{\partial x \partial y} \sin \alpha \cos \alpha\right]^{2} \\
& +\gamma_{A B}\left(\frac{\partial^{2} w}{\partial x \partial y}\right)^{2}+\gamma_{A C}\left(\frac{\partial^{2} w}{\partial x \partial y}\right)^{2}
\end{aligned}
$$

Let now $\rho_{A D}^{\prime}=\rho_{B D}^{\prime}$ we can write:

$$
\begin{aligned}
& \frac{S D_{x}}{2}\left(\frac{\partial^{2} w}{\partial x^{2}}\right)^{2}=\rho_{A B}^{\prime}\left(\frac{\partial^{2} w}{\partial x^{2}}\right)^{2}+\rho_{A D}^{\prime}\left(\frac{\partial^{2} w}{\partial x^{2}}\right)^{2} \cos ^{4} \alpha \\
& \rho_{A B}^{\prime}=\frac{S D_{x}}{2}-\rho_{A D}^{\prime} \cos ^{4} \alpha \\
& \frac{S D_{y}}{2}\left(\frac{\partial^{2} w}{\partial y^{2}}\right)^{2}=\rho_{A C}^{\prime}\left(\frac{\partial^{2} w}{\partial y^{2}}\right)^{2}+\rho_{A D}^{\prime}\left(\frac{\partial^{2} w}{\partial y^{2}}\right)^{2} \sin ^{4} \alpha \\
& \rho_{A C}^{\prime}=\frac{S D_{y}}{2}-\rho_{A D}^{\prime} \sin ^{4} \alpha \\
& S D_{1} \frac{\partial^{2} w}{\partial x^{2}} \frac{\partial^{2} w}{\partial y^{2}}=2 \rho_{A D}^{\prime} \sin ^{2} \alpha \cos ^{2} \alpha \frac{\partial^{2} w}{\partial x^{2}} \frac{\partial^{2} w}{\partial y^{2}} \\
& \rho_{A D}^{\prime}=\frac{S D_{1}}{2} \frac{1}{\sin ^{2} \alpha \cos ^{2} \alpha}
\end{aligned}
$$

$$
\gamma_{A B}+\gamma_{A C}=2 S D_{x y}-4 \rho_{A D}^{\prime} \sin ^{2} \alpha \cos ^{2} \alpha
$$

Replace the value of $\rho_{A D}^{\prime}$ in the different equations, we have then:

$$
\begin{gathered}
\rho_{A B}^{\prime}=\frac{S D_{x}}{2}-\frac{S D_{1}}{2} \cot ^{2} \alpha ; \\
\rho_{A C}^{\prime}=\frac{S D_{y}}{2}-\frac{S D_{1}}{2} \tan ^{2} \alpha ; \\
\rho_{A D}^{\prime}=\rho_{C B}^{\prime}=\frac{S D_{1}}{2} \frac{1}{\sin ^{2} \alpha \cos ^{2} \alpha} ;
\end{gathered}
$$




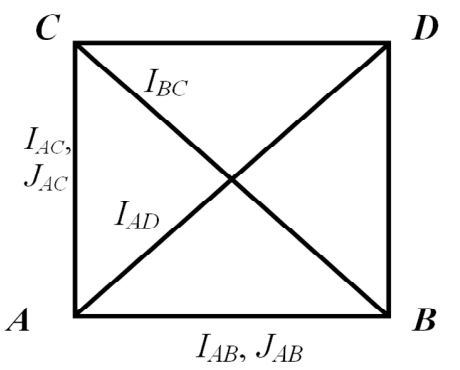

Figure 6. ERC «square with diagonals».

$$
\gamma_{A B}+\gamma_{A C}=2 S\left(D_{x y}-D_{1}\right)
$$

We know that: $\rho_{A B}^{\prime}=E_{l} I_{A B} a, \rho_{A C}^{\prime}=E_{t} I_{A C} b$, $\rho_{A D}^{\prime}=E_{l} I_{A D} l, \gamma_{A B}=G_{l t} J_{A B} a$ and $\gamma_{A C}=G_{l t} J_{A C} b$

Here $I_{i j}$ and $J_{i j}$ denote respectively the second and polar moments of the cross section area of the beam "ij".

By substituting these values in (36), it comes then:

$$
\begin{gathered}
I_{A B}=k \frac{b h^{3}}{24}\left(1-v_{t l} \cot ^{2} \alpha\right) \\
I_{A C}=k \frac{a h^{3}}{24}\left(1-v_{l t} \tan ^{2} \alpha\right) \\
I_{A D}=k \frac{a b h^{3}}{24 l}\left(\frac{v_{t l}}{\sin ^{2} \alpha \cos ^{2} \alpha}\right) \\
J_{A B}=J_{A C}=\frac{a b h^{3}}{6(a+b)}\left(1-k \frac{v_{t l} E_{l}}{G_{l t}}\right) .
\end{gathered}
$$

\section{Static Validation}

\subsection{Simply Supported Plate Submitted to Uniform Distributed Load (Figure 7)}

Consider that the plate has the following geometrical and material properties: length $L=1 \mathrm{~m}$; width $l=0.8 \mathrm{~m}$, and thickness $h=1 \mathrm{~mm}$, longitudinal and transversal Young's moduli: $E_{1}=11.10^{9} \mathrm{~Pa}$ and $E_{t}=11.10^{6} \mathrm{~Pa}$, Poisson's ratio: $v_{l t}=0.3$, Coulomb's coefficient: $G_{l t}=$ $40.10^{6} \mathrm{~Pa}$. That plate is submitted to a uniform constant load $q_{0}=1 \mathrm{~Pa}$.

The theoretical transverse displacement solution of this orthotropic plate problem at any given point with coordinates $(x, y)$ is given in [6] page 59 as

$$
w(x, y)=\sum_{n=1}^{\infty} \sum_{m=1}^{\infty} W_{m n} \sin \left(\frac{m \pi x}{L}\right) \sin \left(\frac{n \pi y}{l}\right)
$$

with

$$
W_{m n}=\frac{q_{m n}}{\left\{\frac{\pi^{4}}{a^{4}}\left[D_{11} m^{4}+2\left(D_{12}+2 D_{66}\right) m^{2} n^{2} r^{2}+D_{22} n^{4} r^{4}\right]\right\}}
$$

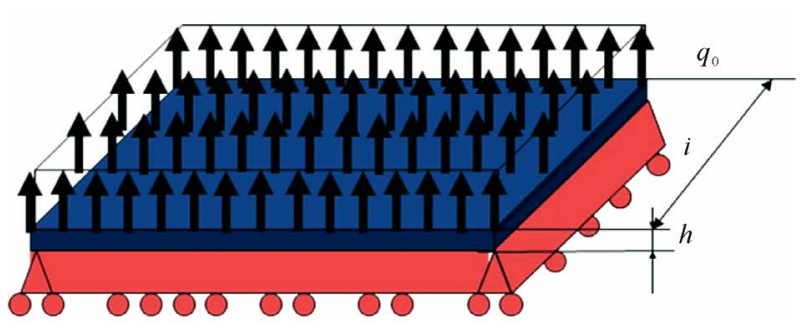

Figure 7. Simply supported plate submitted to uniform distributed load.

$r=L / l$ and

$$
q_{m n}=\frac{16 q_{0}}{m n \pi^{2}} \quad \text { for } \quad m, n=1,3,5, \cdots
$$

The deformed shape obtained after loading is given in Figure 8.

A comparison between the theoretical deformation and the one given by the ET, along the two median lines, $X=$ 0.5 and $Y=0.4$, is shown in Figure 9 .

To evaluate the accuracy of this method, a comparison with the FEM software Patran/Nastran is done for the same size of the mesh and number of nodes as in ET. Quadratic elements are chosen to mesh the thin plate structure in Patran/Nastran. The different results are resumed in Table 1, and compared to the theoretical displacement in the plate center $w_{\text {theoric }}=0.0142368 \mathrm{~m}$. While a better accuracy is reached with the FEM method, as expected, very good agreements are observed between the FEM and ET and even the accuracy with the later still remains good for relatively coarse mesh sizes. Moreover, the convergence to the theoretical solution is also observed with increasing the mesh density.

\subsection{Simply Supported Plate Submitted to Concentrated Load Applied in Its Center}

Let us consider the previous thin plate is now loaded at its center by a concentrated force of magnitude $P=0.01$ N.

The theoretical expression of the displacement solution given in (26) and (27) stay the same, except that

$$
q_{m n}=\frac{4 P}{L l} \sin \left(m \pi \frac{x_{0}}{L}\right) \sin \left(n \pi \frac{y_{0}}{l}\right)
$$

The deformation caused by this load is represented in Figure 10. The related deformation obtained by the ET approach is represented on Figure $\mathbf{1 1}$ and a comparison with the theoretical solution is performed along the two median lines in Figure 11.

The Table 2 provides the related numerical values with in addition the results obtained by the FEM theory obtained for the same size mesh, (same number of nodes). The calculation of the theoretical displacement in the 


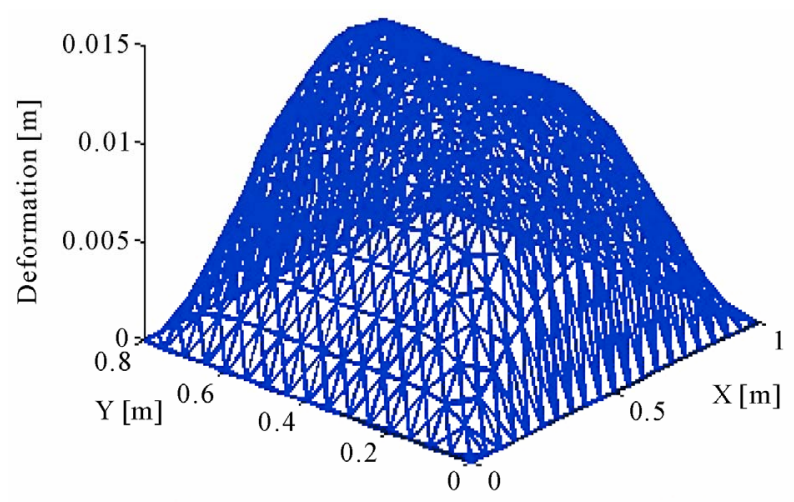

Figure 8. Deformation under a uniform distributed load.

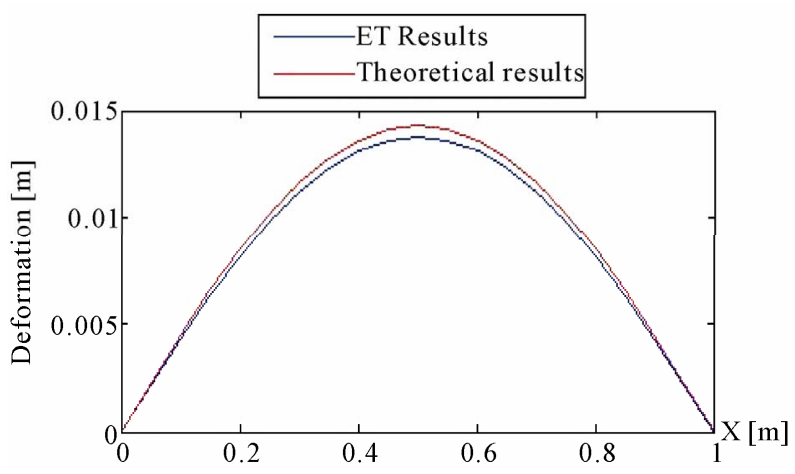

(a)

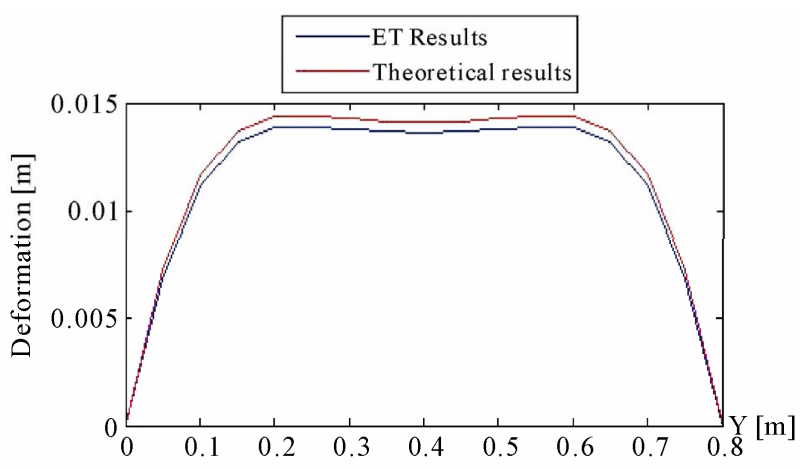

(b)

Figure 9. (a) Superposition of the median lines displacements along the " $X$ " axis; (b) Superposition of the median lines displacements along the " $Y$ " axis.

Table 1. Comparison of the central deformation for a simply supported plate under a uniform loading.

\begin{tabular}{ccccc}
\hline $\begin{array}{c}\text { mesh } \\
\text { size }\end{array}$ & $\begin{array}{c}\text { FEM } \\
\text { deformation } \\
{[\mathrm{m}]}\end{array}$ & $\begin{array}{c}\text { EM } \\
\text { deformation } \\
{[\mathrm{m}]}\end{array}$ & $\begin{array}{c}\text { Error } \\
\text { FEM (\%) }\end{array}$ & $\begin{array}{c}\text { Error ET } \\
(\%)\end{array}$ \\
\hline $10 \times 8$ & $1.405 \mathrm{E}-02$ & $1.340 \mathrm{E}-02$ & $-1.34 \%$ & $-4.59 \%$ \\
$20 \times 16$ & $1.422 \mathrm{E}-02$ & $1.378 \mathrm{E}-02$ & $-0.12 \%$ & $-3.11 \%$ \\
$30 \times 24$ & $1.425 \mathrm{E}-02$ & $1.390 \mathrm{E}-02$ & $0.08 \%$ & $-2.46 \%$ \\
\hline
\end{tabular}

plate center gave $w_{\text {theoric }}=0.00132144758109 \mathrm{~m}$. Once again, we observe that the two methods converge to the theoretical solution, when we increase the mesh size. Unlike the first example, we observe here (as the main result of the statics section) that the ET provides better results than those of the FEM.

\section{Application of the Equivalence Theory in Dynamics}

In statics we have observed that the ET provides very good results, which are in agreement with the literature [1-3], and we have obtained the same conclusions with anisotropic plates. Now we will test this method in dynamics.

In our study we consider the mass conservation of the system. By equating the overall masses of the continuous plate and lattice models, and supposing that a homogeneous mass density in the lattice structure, we have:

$$
\rho_{P} \cdot L \cdot l \cdot h=\rho \sum_{e=1}^{N} A_{e} \cdot a_{e} \text { and so } \rho=\frac{\rho_{P} \cdot L \cdot l \cdot h}{\sum_{e=1}^{N} A_{e} \cdot a_{e}}
$$

with $A_{e}, a_{e}$ are respectively the cross section and length of the $e^{\text {th }}$ beam, $\mathrm{N}$ is the number of the beams, $\rho$ the mass density of the beams, $\rho_{p}$ the mass density of the plate, $L, l$ and $h$ are respectively the length, width and thickness of the plate.

To calculate the inertial moment of the beams we have to precise a shape for the cross sections. We choose circular ones, having the section from the static equivalence; we calculate first the beam cross section radii $R_{e}=\sqrt{A_{e} / \pi}$ and their inertial moment

$$
I_{e}=\frac{\rho \cdot l_{e} \cdot A_{e} \cdot R^{2}}{2}
$$

\section{Example: Clumped Rectangular Plate}

\subsection{Frequencies}

The plate considered here is the same as in the first example, we have just clamped it in its four sides.

In dynamics we preferred to compare the ET results to the FEM ones. We have choose a mesh of $200 \times 160$ elements in Nastran/Patran software, and we defined the plate as a shell element, these was to approximate better the real solutions.

In what follows we will show the first hundred "100" frequency values obtained for different mesh sizes, and for two representations of the mass matrix, the first is the consistent mass matrix, which is the same expressed in the classic "FEM" formulation, and the second one is the lumped mass matrix, whish considered that the mass and 


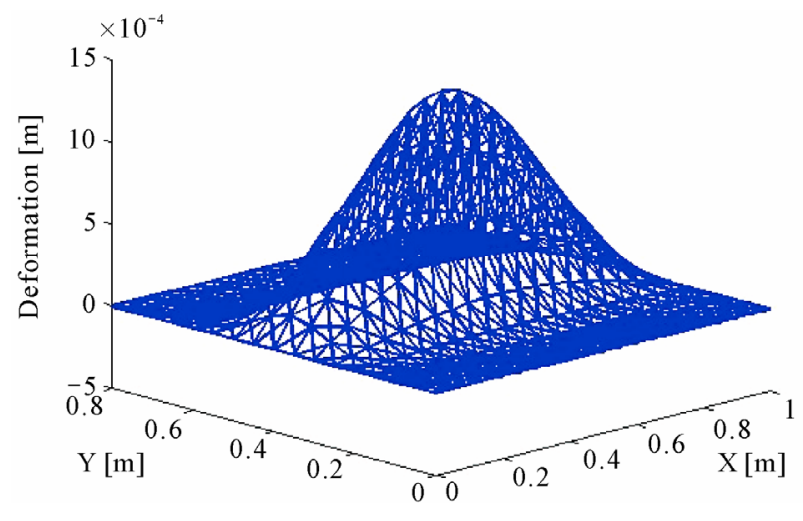

Figure 10. Deformed shape under a before concentred centred load.

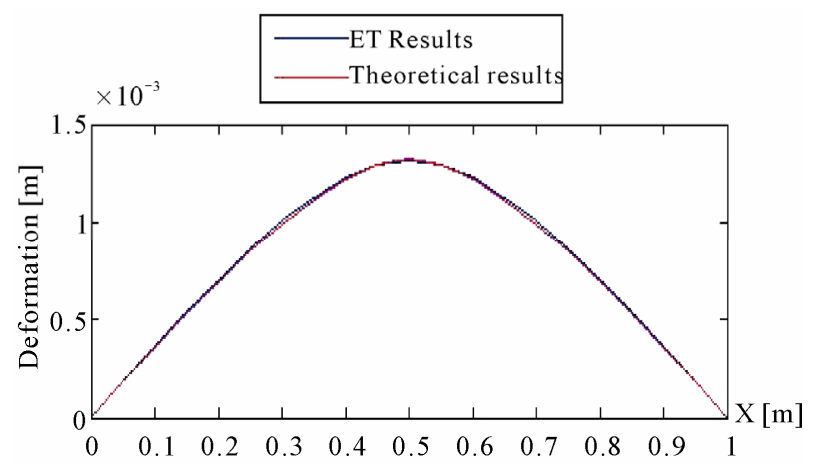

(a)

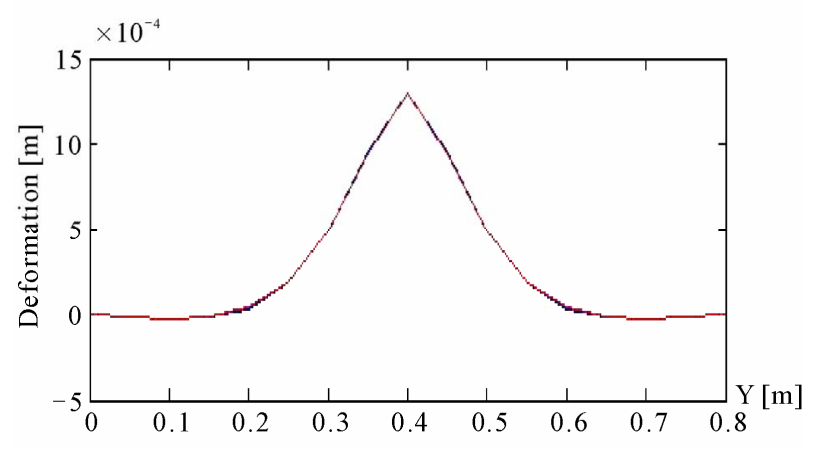

(b)

Figure 11. Superposition of the results among the median lines $(\mathrm{A}-\mathrm{X}=0.5 ; \mathrm{B}-\mathrm{Y}=\mathbf{0 . 4})$.

Table 2. Comparison of the central deformation for a simply supported plate under a concentrated load applied in its center.

\begin{tabular}{ccccc}
\hline $\begin{array}{c}\text { mesh } \\
\text { size }\end{array}$ & $\begin{array}{c}\text { FEM } \\
\text { deformation } \\
{[\mathrm{m}]}\end{array}$ & $\begin{array}{c}\text { EM } \\
\text { deformation } \\
{[\mathrm{m}]}\end{array}$ & $\begin{array}{c}\text { Error FEM } \\
(\%)\end{array}$ & $\begin{array}{c}\text { Error ET } \\
(\%)\end{array}$ \\
\hline $10 \times 8$ & $1.417 \mathrm{E}-03$ & $1.291 \mathrm{E}-03$ & $9.78 \%$ & $-2.34 \%$ \\
$20 \times 16$ & $1.367 \mathrm{E}-03$ & $1.321 \mathrm{E}-03$ & $3.49 \%$ & $-0.02 \%$ \\
$30 \times 24$ & $1.348 \mathrm{E}-03$ & $1.325 \mathrm{E}-03$ & $1.77 \%$ & $0.24 \%$ \\
\hline
\end{tabular}

the inertial moment is equitably distributed in the nodes of the beams. Moreover we will have for the translation the half mass working in the three direction " $u, v, w$ " and the half inertial moment working in the three rotating directions " $\theta_{x}, \theta_{y}, \theta_{z}$ " . Note that the bending isn't considered here, this approach is the same as the Patran/Nastran one for the lumped mass matrix.

Figure 12 shows the first hundred eigenvalues of the plate obtained by the ET and confronts them with the values obtained by finite element method. We see very clearly that when the mesh size in the ET is increasing, the curve obtained by this method tends to approximate that obtained by FEM. We also note that even with a coarse size mesh, the first eigenvalues are very close to those obtained by the FEM. The evolution of the relative discrepancy between the ET results versus the FEM ones is plotted in Figure 13. In fact, we observe that the first five " 5 " frequencies obtained are very close to those obtained by FEM even when we use small size mesh. The convergence as the number of elements is increasing is very remarkable, we note that the first hundred " 100 " frequencies are obtained with a maximum error of $25 \%$ for a mesh of $(20 \times 16)$ to $12 \%$ for a mesh of $(30 \times 24)$. The convergence is also observed in the case a distributed (lumped) mass matrix. Indeed we notice that with the mesh size of $(20 \times 16)$ we have a maximum error of $86 \%$, this error is reduced to $16 \%$ for a mesh size of $(30$ $\times 24)$. We clearly note that the best results are obtained for a consistent weight distribution.

\subsection{Mode Shapes}

Figure 14 illustrates the first ten mode shapes obtained by the ET approach. These mode shapes are exactly the same than those found in literature [7].

\section{Conclusions}

Heretofore the equivalence theory has been expressed only for isotropic static problems. The formulation is based in equating the strain energies of two systems. The researchers $[1,3,4]$ were interested by the calculation of deformations, bending moments, convergence with increasing mesh size. They found that in statics this method is very robust, it gives very good results with small mesh sizes and the results are converging when we increase the mesh size.

This method may provide a sheap, sufficient reliable, and convient approach to treat complex structural systems as the computer storage requirement and running times are small compared with those of other numerical methods.

In order to demonstrate the validity of the procedure 

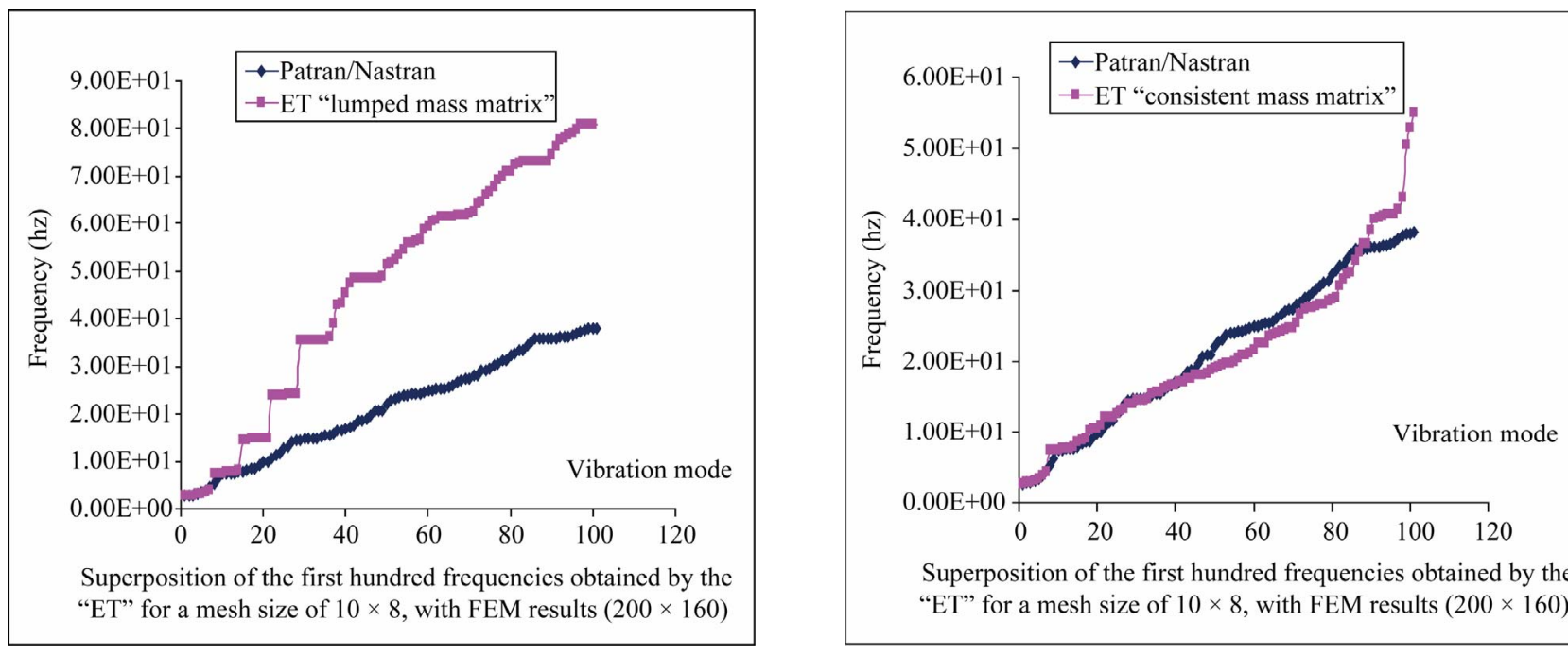

Superposition of the first hundred frequencies obtained by the "ET" for a mesh size of $10 \times 8$, with FEM results $(200 \times 160)$
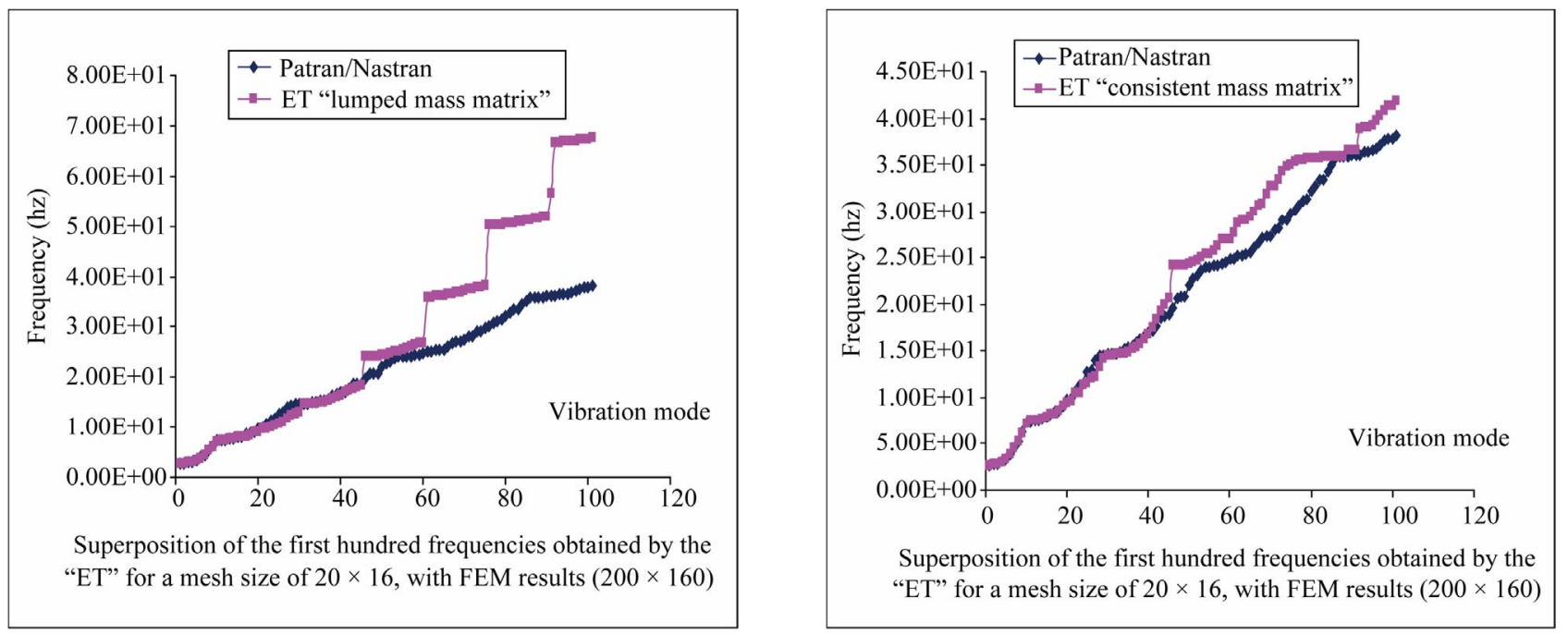

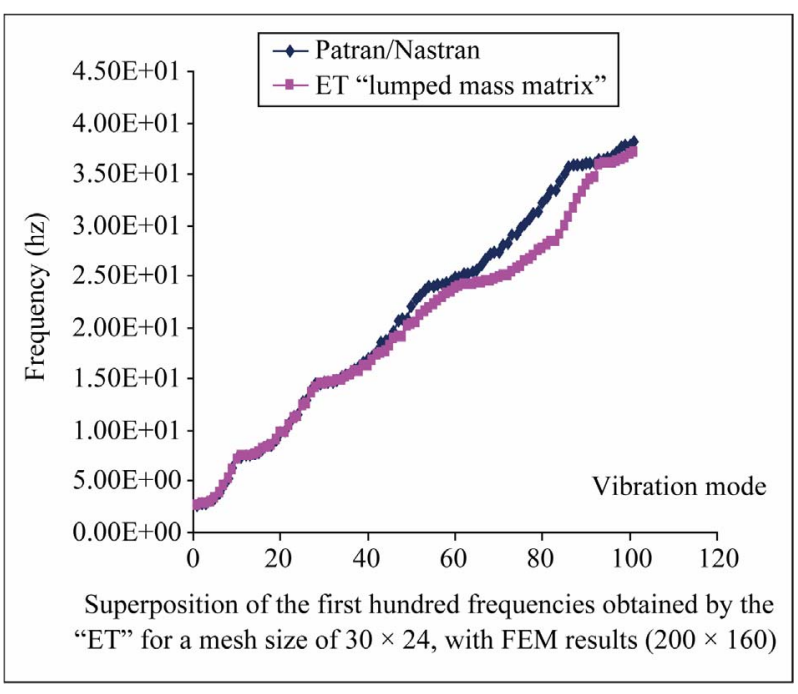

(a)

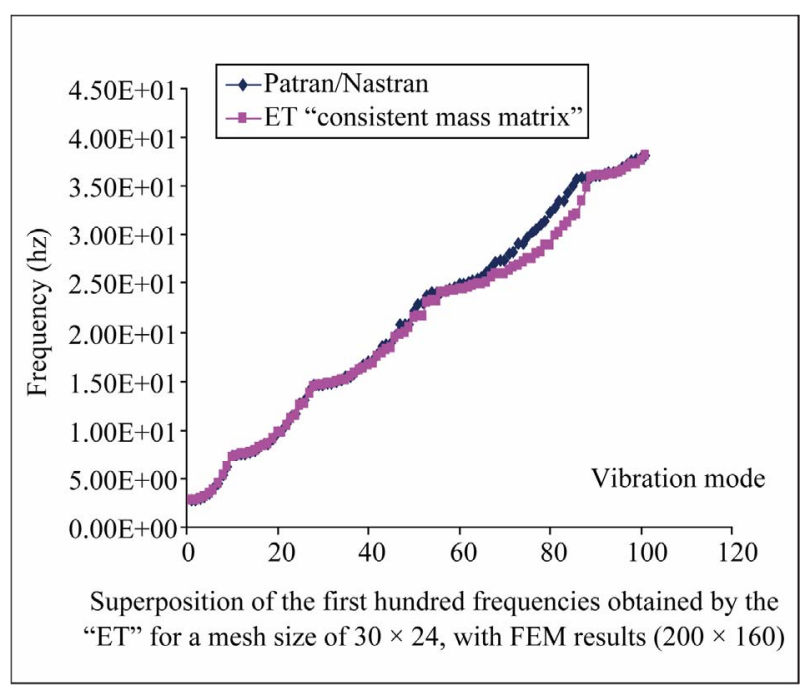

(b)

Figure 12. Comparison of the first hundred frequencies obtained by the ET and those provided by the software Patran/ Nastran. (a) Lumped mass matrix; (b) Consistent mass matrix. 

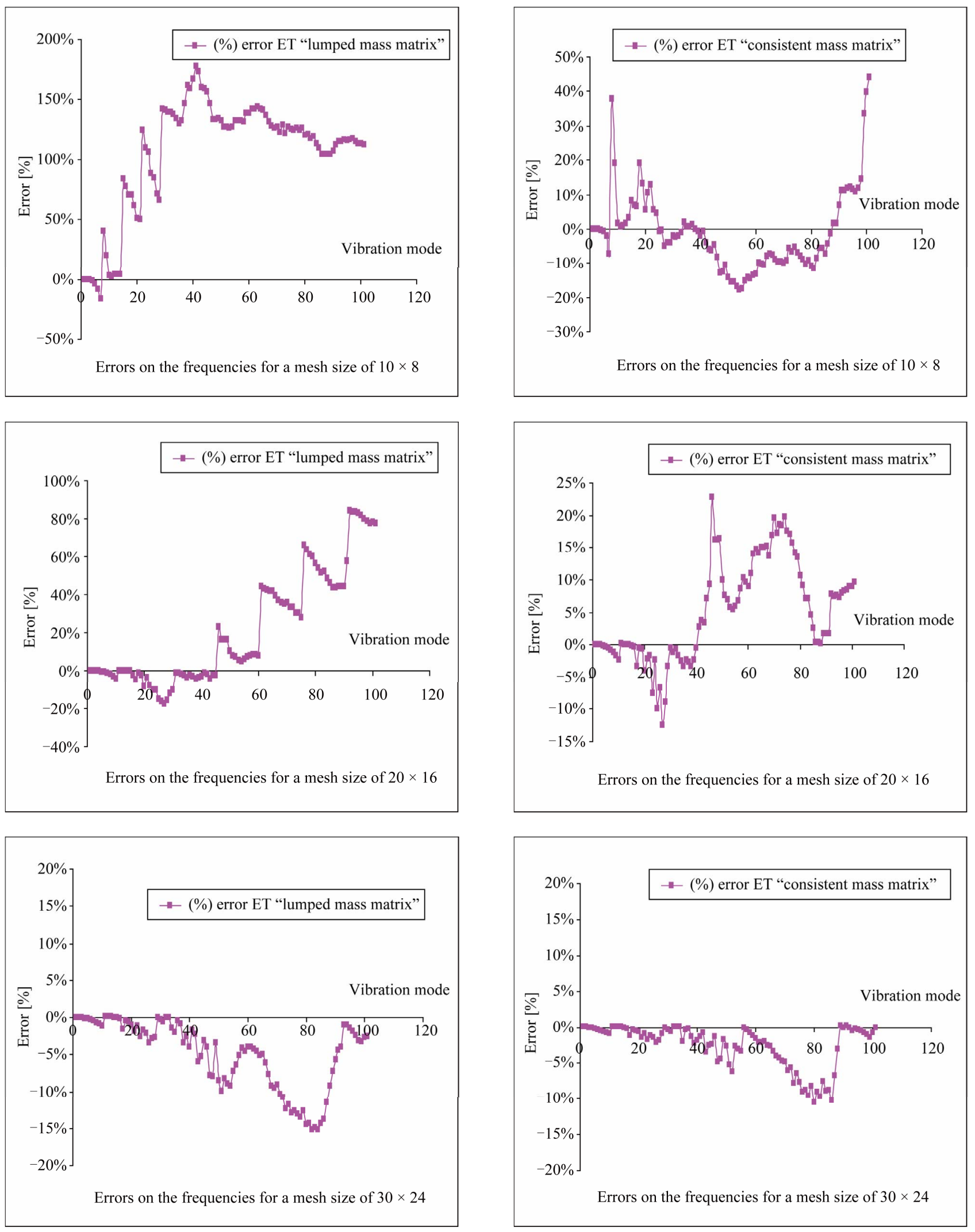

(a)

(b)

Figure 13. Errors obtained for the first hundred frequencies. (a) Lumped mass matrix; (b) Consistent mass matrix. 

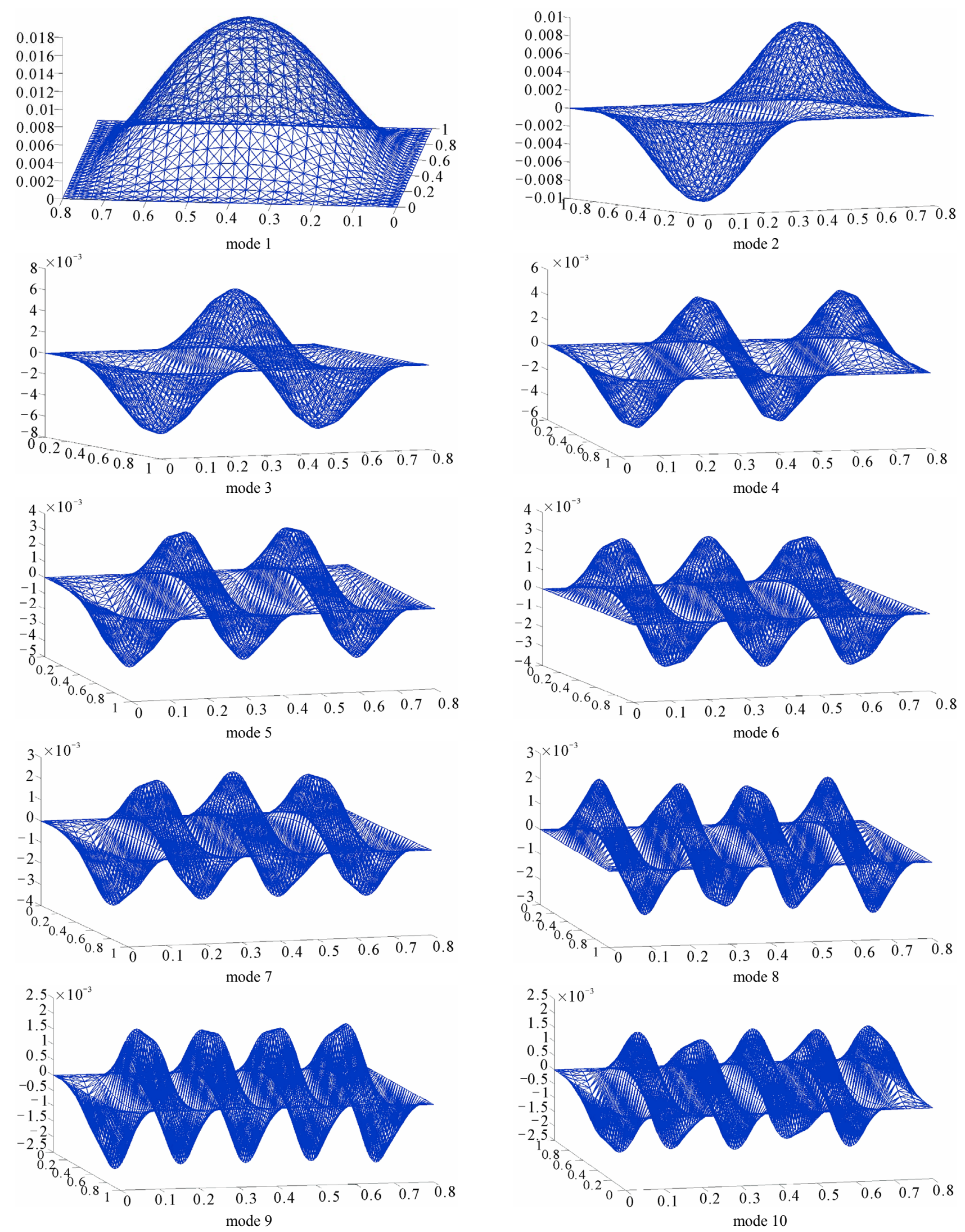

Figure 14. Mode shapes of orthotropic clumped plate. 
for anisotropic static problems, two example problems, relevant to plane stress (thin plates) were examined by using the ET approach and the FEM with different grid arrangement. The results are in good agreement with the analytical solutions even in the case of a small number of nodes (coarse mesh). Most importantly, we have also noted that these results are very near and even in some cases better than those predicted by the FEM.

To extend this procedure to dynamics, we have just formulated in addition a simple mass conservation rule, and choose a circular form for our beams. In order to validate this procedure, we compared the results to those of the FEM with a relatively fine mesh. We have not made a comparison with analytical solutions because this latter proposes approximate solutions concerning only the bending of the plates, while the FEM gives the different vibration modes (bending, torsion, traction...). We considered the problem of a thin clamped plate, and found that the results are in good agreement with the FEM solutions. We note also that the formulation of the mass as a consistent one gave best results; this results are explained by the fact that the mass is well distributed in the ERC and approaches the homogeneous distribution in the real cell. The lumped representation of the mass is found to be convenient in the high computational speed, indeed the mass matrixes are diagonals, and so easier to invert, moreover we observe that its results remain close to those of a consistent formulation. Thus the user can be free to choose between very accurate results and high computational speed.

A future work could be to deal with 3D problems. We have created the connectivity table of different 3D
"ERC", and begin the solving of the problem concerning the expression of the stiffness matrix in 3D. The analysis performed for the 2D models can be extended to the 3D ones and also addresses other problems like fatigue and blucking.

\section{References}

[1] E. ABSI, "La Theorie des Equivalences et Son Application a l'Etude des Ouvrages d'Art," Série: Théories et Méthodes de Calcul, Annales de l'Institut Technique du Bâtiment et des Travaux Publics, Supplément No. 298, Octobre 1972.

[2] M. Haddad, "Application de la Methode des Equivalences en Dynamique," Rapport de Stage Master2 Recherche, l'Institut Supérieur de l'Aéronautique et de l'Espace (ISAE), Toulouse, 7 February 2010.

[3] S. Vegas, "Application de la Theorie des Equivalences a l'Etude d'Une Dalle Biaise," PhD Thesis, University Paul Sabatier de Toulouse, 11 June 1976.

[4] G. M. Cucchi, "Elastic-Static Analysis of Shear Wall/Slab-Frame Systems Using the Framework Method," Pergamon, 30 June 1993.

[5] S. Timochenko, S. W. Kreiger, "Théorie des Plaques et Coques," Librairie Polytechnique CH, Beranger, 1961.

[6] S. Abrate, "Inpact in Composite Structures," Cambridge University Press, Cambridge, 1998, pp. 59-61. doi:10.1017/CBO9780511574504

[7] W. Leissa, "Vibrations of Plates," Ohio State University Columbus, Ohio, Edition Scientific and Technical Information Division, Office of Technology Utilization, National Aeronautics and Space Administration, Washinton, DC, 1969. 